\section{A) Check for updates}

Cite this: Nanoscale, 2020, 12, 16315

\title{
Short-term angiotensin II treatment regulates cardiac nanomechanics via microtubule modifications $\uparrow$
}

\author{
Pamela Swiatlowska, (D) ${ }^{a}$ Jose L. Sanchez-Alonso, ${ }^{a}$ Catherine Mansfield, ${ }^{a}$ \\ Denis Scaini, ${ }^{b, c}$ Yuri Korchev, ${ }^{\text {b,d }}$ Pavel Novak ${ }^{b, e}$ and Julia Gorelik*a
}

\begin{abstract}
Mechanical properties of single myocytes contribute to the whole heart performance, but the measurement of mechanics in living cells at high resolution with minimal force interaction remains challenging. Angiotensin II (AngII) is a peptide hormone that regulates a number of physiological functions, including heart performance. It has also been shown to contribute to cell mechanics by inducing cell stiffening. Using non-contact high-resolution Scanning Ion Conductance Microscopy (SICM), we determine simultaneously cell topography and membrane transverse Young's modulus (YM) by a constant pressure application through a nanopipette. While applying pressure, the vertical position is recorded and a deformation map is generated from which YM can be calculated and corrected for the uneven geometry. High resolution of this method also allows studying specific membrane subdomains, such as Z-grooves and crests. We found that short-term Angll treatment reduces the transversal YM in isolated adult rat cardiomyocytes acting via an AT1 receptor. Blocking either a TGF- $\beta 1$ receptor or Rho kinase abolishes this effect. Analysis of the cytoskeleton showed that Angll depletes microtubules by decreasing long-lived detyrosinated and acetylated microtubule populations. Interestingly, in the failing cardiomyocytes, which are stiffer than controls, the short-term Angll treatment also reduces the YM, thus normalizing the mechanical state of cells. This suggests that the short-term softening effect of Angll on cardiac cells is opposite to the wellcharacterized long-term hypertrophic effect. In conclusion, we generate a precise nanoscale indication map of location-specific transverse cortical YM within the cell and this can substantially advance our understanding of cellular mechanics in a physiological environment, for example in isolated cardiac myocytes.
\end{abstract}

Received 27th March 2020,

Accepted 27th June 2020

DOI: $10.1039 / \mathrm{d} 0 \mathrm{nr} 02474 \mathrm{k}$

rsc.li/nanoscale
Work by Barnabei and Metzger demonstrates that cellular and organ compliance can differ in pathological settings. ${ }^{4}$ Therefore, to understand a particular disease mechanism, different organizational levels should be examined. Cells, due to their complex organization, have a collection of different components, each with their own mechanical properties, that in total represent the mechanical behavior of the cell. Several features contribute to the cellular response to mechanical stimuli, among them the cell transversal stiffness measured as the Young's Modulus (YM) has become one of the prevailing parameters studied in recent years. YM is defined as the amount of force per unit area (referred to as stress) per relative extension (referred to as strain) $\left[\mathrm{YM} ; \mathrm{Pa}=\mathrm{N} \mathrm{m}^{-2}\right]$ or, in simple words, YM is a measure of the resistance of the material to deformation.

For example, a substantial increase in Young's modulus (YM) of myocytes is observed from the embryonic to the postnatal stages of development. ${ }^{5}$ Mechanical stimulation by itself has been reported to have a significant role in the develop- 
ment of cardiomyocytes differentiated from human induced pluripotent stem cells, as substrate elasticity as well as confinement of the cell shape resulted in improved contractility and electrophysiology and a better mitochondrial arrangement, or even increased the presence of transverse tubules. ${ }^{6}$ The mechanical properties of adult cardiomyocytes, including the passive properties of the membrane such as YM, have been associated with the changes in cell electrophysiology and the elements of the mechanotransduction pathway. ${ }^{7-9}$ On the other hand, in the case of altered mechanical load in the adult heart, which is common under pathological conditions, cardiac myocytes acquire either hypertrophic or atrophic phenotypes in order to meet the current mechanical needs, ${ }^{10,11}$ with corresponding changes in the mechanical properties of the cells. However, it is still poorly understood whether an altered YM is the direct cause of a disease or simply a consequence of the changes taking place in the disease.

Angiotensin II (AngII) is a peptide hormone mainly responsible for vasoconstriction and maintaining the water-salt equilibrium $^{12}$ with a range of other effects in different organs. Long term AngII treatment has been shown to have adverse effects on the myocardium by inducing cardiac hypertrophy that is associated with increased YM. However, a study by Leite-Moreira et al. has shown that in rabbit heart papillary muscle, acute AngII exposure evokes a change in passive length-tension indicating a reduced longitudinal YM. ${ }^{13}$ This change has been demonstrated to be dependent on protein kinase $\mathrm{C}$ (PKC) and the $\mathrm{Na}^{+} / \mathrm{H}^{+}$exchanger. Overall, the molecular mechanism on how AngII acutely acts on cardiomyocyte mechanics is still not clear. More insight into this signaling cascade is needed to understand the time point and changes that drive the switch from a favorable pathway to a detrimental one.

To date, different techniques, such as micropipette aspiration, optical tweezers, or atomic force microscopy (AFM) to name a few, have been employed to measure YM in biological samples. Briefly, micropipette aspiration is based on a simple principle of single cell aspiration by a negative pressure, where cell deformation is observed using an optical microscope and cell's YM is simultaneously measured by employing theoretical models. ${ }^{14,15}$ Optical tweezers use a laser beam and generate trapping force to immobilize the cells to measure mechanical properties. $^{16,17}$ Atomic Force Microscopy (AFM) has been the most frequently used technique to image the topography and resolve the mechanical properties of cells as it allows acquiring nanoscale structures using a probe mounted on the tip of a flexible cantilever. ${ }^{13,18,19}$ Nevertheless, each technique has its limitations such as time-consuming measurements, difficulty in manufacturing instruments, long exposure time to high intensity light affecting cell enzymatic reactions, lack of selectivity or high sample indentation in direct contact mode that makes it difficult to assess the sample-probe interaction.

A previous work from our group has shown that failing cardiomyocytes are susceptible to abnormal calcium release after applying mechanical stimuli and that could lead to arrhythmias. Nanomechanical changes at the cortical membrane, including membrane stiffness, have been suggested to be factors responsible for the electrical activity. ${ }^{20}$ Measurements of the transverse YM could reveal the significance of this passive property for cell physiology.

Recently, Scanning Ion Conductance Microscopy (SICM) has been employed to measure the YM of living cells. ${ }^{21,22}$ Using this technique, mechano-Scanning Ion Conductance Microscopy (mechanoSICM), we have shown that the microtubular network affects the transverse YM of cardiomyocytes in the heart with altered mechanical load. ${ }^{23}$

In this work, to reveal the acute effect of AngII on cardiac myocytes, we used mechanoSICM and found that short-term AngII treatment generates a softening effect (reduced YM) in isolated adult rat cardiomyocytes, which is dependent on the Angiotensin Type 1 (AT1) receptor and indirectly mediated by transforming growth factor- $\beta 1$ (TGF- $\beta 1$ ) and Rho kinase. Furthermore, investigation of microtubules (MTs) after AngII treatment revealed a significant decrease in acetylated and detyrosinated MT populations; this provides evidence that post-translational modifications (PTMs) that stabilize MTs were the most affected by AngII.

Interestingly, in cells isolated from failing hearts (after 16 weeks of myocardial infarction), which are pathologically stiffer than control cells, AngII also has a softening effect. This proves mechanoSICM to be a suitable method for analyzing cell mechanics in cultured cells.

\section{Results}

\section{Adaptation of scanning ion conductance microscopy to measure Young's modulus in live samples}

Scanning Ion Conductance Microscopy (SICM) is a microscopy technique by which one can obtain the topography map of an analyzed sample of living cells in non-contact mode with a 10-30 nm resolution. ${ }^{24,25}$ Briefly, the working principle is based on the ion flow through the glass nanopipette. A decrease in the ion current flow is observed when the nanopipette approaches the sample surface. To preserve a sampleprobe distance of at least equal to the inner pipette radius, the feedback system stops the approach when the drop in current through the pipette is typically between $0.3 \%$ and $1 \%$. The position of the $z$-axis actuator is recorded when the current achieves this reduction. By scanning the sample in $X$ and $Y$ dimensions, a topographical image of the surface of the sample is obtained.

In mechanoSICM, the system has been modified in order to map the YM simultaneously with the topography area. For this, a small, highly localized force is imposed onto the cell surface via the nanopipette by applying a $15 \mathrm{kPa}$ aerostatic pressure through a syringe connected through a side port on a pipette holder. The local value of YM, which reflects cell transversal stiffness, can be estimated during scanning and displayed as a map. ${ }^{21}$ The pressure application propels a jet of solution out of the pipette tip which exerts the mechanical force on the cell membrane. 


\section{Topographical slope correction}

Adult cardiac myocytes are elongated cells with clearly defined membrane structures. Along the surface of cardiomyocytes, deep invaginations of the sarcolemma can be seen and are the openings of the internal system of transverse tubules (TT); these structures are regularly distributed to form a striation pattern of Z-grooves which are separated by $2 \mu \mathrm{m}$ from each other, reflecting the internal distribution of the sarcomere structures. The curved areas of the sarcolemma between Z-grooves and crests can be clearly observed by Transmission Electron Microscopy. Therefore, the cardiomyocyte surface is far from being flat, and this can affect the YM measurements. It has been reported that the sample slope affects the way the ionic current drops when the nanopipette approaches the sample. ${ }^{26}$ Thatenhorst et $a .^{26}$ have shown that the pipette current drops slower with distance when the pipette is approaching the inclined sample surface than when it is approaching a perfectly horizontal surface. This effect on the current-distance dependence can be corrected simply by measuring the tip-sample distance in the direction perpendicular to the inclined surface, instead of along the nanopipette. The mapping of YM using liquid flow from the nanopipette utilizes the fact that when a pipette approaches a soft surface the current drops slower with distance than when it approaches a stiff surface. ${ }^{21}$ In our implementation of the method, the YM value is estimated from the distance that the nanopipette travels while the current drops between $1 \%$ and $2 \%$. The distance will be bigger for a softer surface than for a stiffer surface. Taking into account the work by Thatenhorst et al. ${ }^{26}$ one can assume that the slope of the current-distance relationship will be in reality affected by both the local topography slope and the stiffness of the material, which may cause inclined areas to appear softer than horizontal areas. To compensate for the reduction in YM due to the effect of the local slope on the distance measurement, all distances travelled by the nanopipette would be recalculated into distance normal to the surface (ESI Fig. $1 \dagger$ ).

$$
d_{\mathrm{n}}=d \times \cos \alpha
$$

Where $d$ is the surface-nanopipette distance measured along the $z$-axis, $d_{\mathrm{n}}$ is the surface-nanopipette distance measured along direction perpendicular to the surface, and $\alpha$ is the angle of the slope. The distance along the surface normal $d_{\mathrm{n}}$ is then used to calculate Young's modulus (see Materials and methods for detailed description).

To confirm the effectiveness of the correction, we have performed an experiment on decane droplets deposited on a plastic culture dish in phosphate buffered solution (ESI Fig. $2 \dagger$ ). The surface of a decane droplet will resist deformation due to surface tension and therefore will appear to the approaching nanopipette as a material possessing an apparent Young's modulus despite being a liquid. Decane droplets show highly spherical topography but are expected to be homogeneous from the viewpoint of the mechanical properties, so any major decrease in the apparent YM on inclined areas is expected to be mainly due to the slope. The inclined areas of the droplet topography indeed showed markedly reduced apparent YM values (ESI Fig. 2B and E $\dagger$ ). The application of the distance correction markedly reduced the occurrence of extremely low apparent YM values (10-40 Pa, ESI Fig. $2 \mathrm{C}$ and $\mathrm{D} \dagger$ ) and reduced the decrease in apparent $\mathrm{YM}$ on slopes above $10^{\circ}$ by a median value of $7.2 \%$ (mean value of 45.7\%) (ESI Fig. 2E†). When considering the range of slopes typically observed in cardiomyocytes $\left(0-30^{\circ}\right.$, ESI Fig. $\left.1 \mathrm{~B} \dagger\right)$, the correction reduced the decrease in apparent $\mathrm{YM}$ values by a median value of $7.7 \%$ (10.4\% mean value) for slopes between 10 and $30^{\circ}$ (ESI Fig. 2E $\dagger$ ). This suggests that the slope correction has the potential to reduce the effect of the slope on the measurement of the YM for cardiomyocytes.

We would like to stress that the correction procedure presented here corrects only for the errors in the distance measurement which affects the estimation of YM. The effect of the local slope on the liquid flow out of the pipette and the resulting stress imposed by the flow on the sample surface was not considered. Therefore, the measured value of YM on significantly inclined surfaces is likely to represent a combination of a tensile modulus (perpendicular to the surface) and a shear modulus (parallel to the surface). This may partially account for the sustained reduction in apparent YM observed at droplet slopes steeper than $30^{\circ}$ (ESI Fig. $2 \mathrm{E} \dagger$ ), where the viscosity of the droplet in response to shear stress is likely to have an increasingly dominant influence.

\section{The role of angiotensin II in cardiac mechanics}

Previous work from Leite-Moreira suggested that the multifunctional peptide hormone, AngII, can regulate the cardiomyocyte mechanoproperties. ${ }^{13}$ AngII is a well-known hypertrophic factor in long term treatments and tends to increase the cell YM over time. ${ }^{27}$ However, studies on the rabbit papillary muscle and in situ heart models show that after 15 min of treatment with AngII a shift in the length-resting tension relation is observed, which indicates a lower longitudinal YM. ${ }^{13}$ Using mechanoSICM, we aimed to examine whether short-term AngII shows an effect on the transverse YM and further investigate the signaling pathway.

Cellular transverse YM of adult rat cardiomyocytes was measured after 2 hours of incubation with AngII at different concentrations (Fig. 1A). Based on this dose response curve, the concentration of $20 \mu \mathrm{M}$ AngII was chosen for all the following experiments. Although this concentration is higher than what has been reported in vivo, ${ }^{28}$ it is consistent with other in vitro experiments from several groups. ${ }^{29-32}$ We observed an overall and domain-specific decrease in the YM value following the treatment of control cardiomyocytes (AngII Z-groove $1.88 \pm$ $0.14 \mathrm{kPa}$, crest $1.89 \pm 0.18 \mathrm{kPa} v$ s. control Z-groove $2.64 \pm 0.26$ $\mathrm{kPa}$, crest $3.1 \pm 0.33 \mathrm{kPa}, p<0.01$ ) (Fig. $1 \mathrm{~B}$ and C). This softening of the cell surface is due to the activation of AT1 receptors, as losartan (AT1 receptor inhibitor) abolishes the effect of AngII on transverse YM (AngII Z-groove $1.88 \pm 0.1 \mathrm{kPa}$, crest $1.97 \pm 0.14 \mathrm{kPa} v s$. AngII + Los Z-groove $2.99 \pm 0.31 \mathrm{kPa}$, crest $3.26 \pm 0.35 \mathrm{kPa}, p<0.05$ ) (Fig. 1B and C). 
A
B
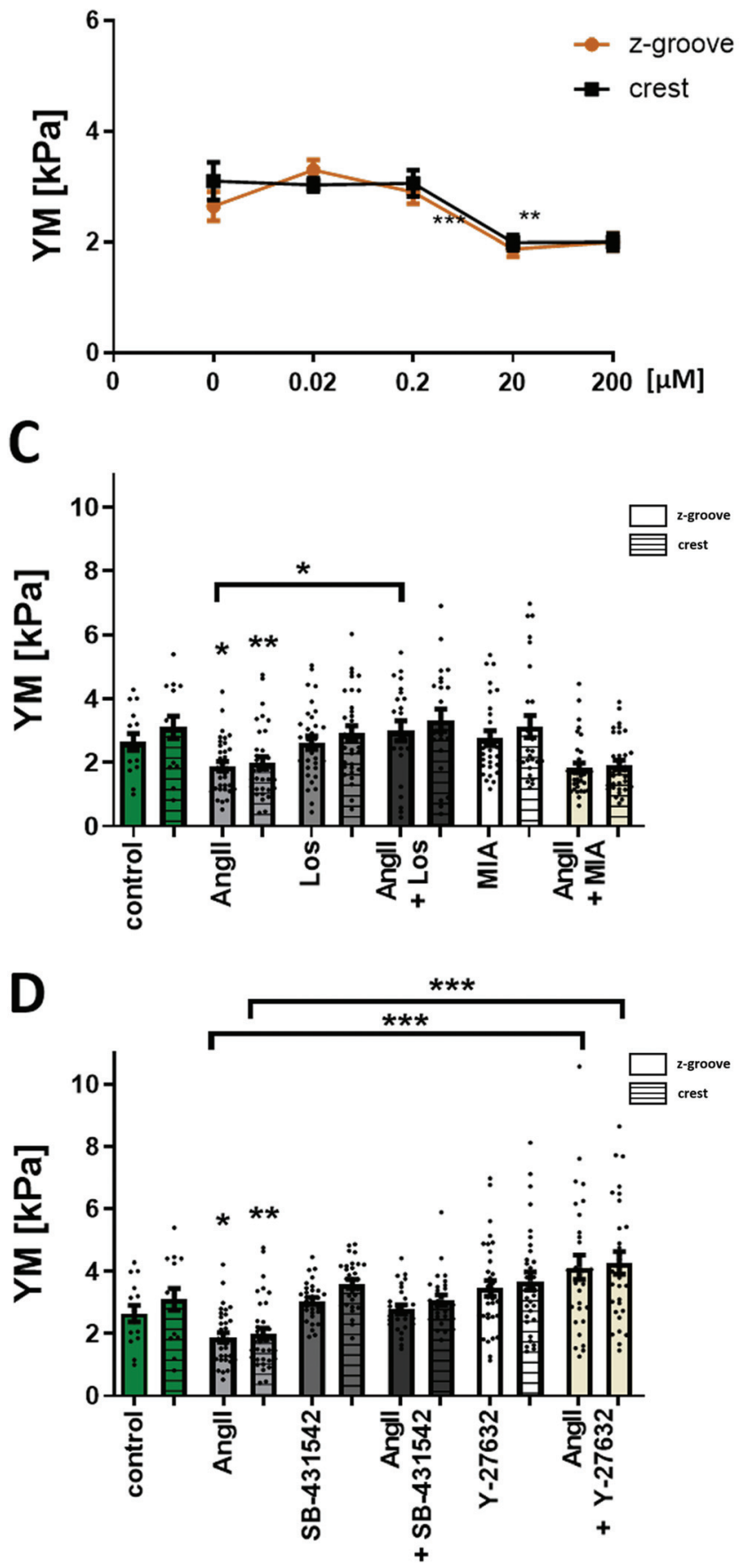
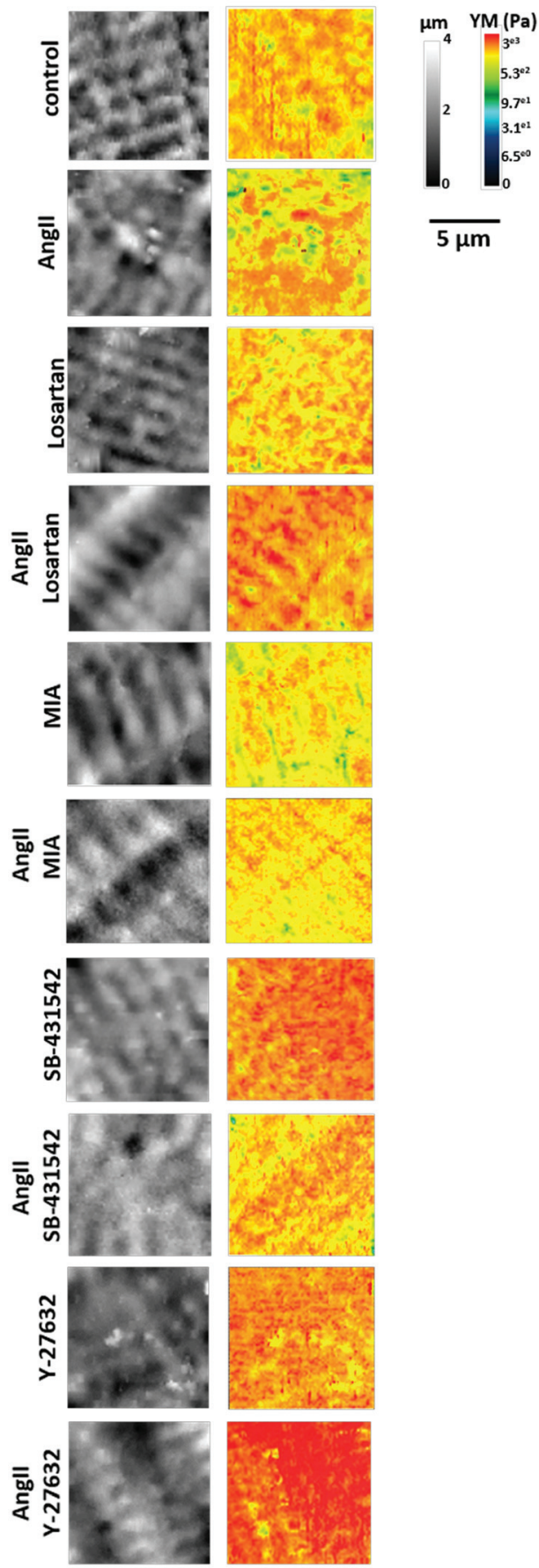

Fig. 1 Angll effect on YM and the mechanism of action. (A) Dose response curve of Angll-treated cells. A significant decrease in YM is observed following $20 \mu \mathrm{M}$ Angll incubation. $N / n=3 / 20-40$. Data presented as mean \pm SEM. ${ }^{* * *} p<0.001$. (B) Representative $10 \mu \mathrm{m} \times 10 \mu \mathrm{m}$ SICM images of topography and YM maps. (C) Quantification of YM after treating the cells with Angll, with the AT1 receptor blocking agent (Losartan), and with the $\mathrm{Na}^{+} / \mathrm{H}^{+}$exchanger inhibitor (MIA). (D) Quantification of YM after treating the cells with the TGFB1 receptor blocking agent (SB-431542) or the Rho kinase inhibitor (Y-27632). $N / n=3 / 15-35$. Data presented as mean $\pm \mathrm{SEM} .{ }^{*} p<0.05,{ }^{* \star} p<0.01,{ }^{* \star *} p<0.001$.

Previously, it has been demonstrated that mechanical alterations are related to acute treatments of AngII, ${ }^{13}$ suggesting the $\mathrm{Na}^{+} / \mathrm{H}^{+}$exchanger and PKC as the target for AngII. To find out whether this mechanism plays a role in the effects we see after acute treatment with AngII, we treated adult rat cardiomyocytes with the $\mathrm{Na}^{+} / \mathrm{H}^{+}$inhibitor $5-(N-$ methyl- $N$-isobutyl)-amiloride (MIA) alone and in combination with AngII. The inhibitor itself did not change YM values, and 
the AngII effect was unaffected by the presence of the inhibitor (AngII + MIA Z-groove $1.83 \pm 0.15 \mathrm{kPa}$, crest $1.9 \pm 0.14 \mathrm{kPa} v s$. AngII, $p>0.05$ ) (Fig. 1B and C), suggesting a lack of involvement in the $\mathrm{Na}^{+} / \mathrm{H}^{+}$exchanger in our experimental conditions.

AngII can also produce its effect through the TGF- $\beta 1$ pathway, as cross talk within the myocardium has been previously established. ${ }^{33-35}$ To test whether AngII interference with TGF- $\beta 1$ signaling could contribute to its effect on the cell's YM, we used a TGF- $\beta 1$ inhibitor, SB-431542. As with Losartan, the inhibitor itself did not change YM values and the AngII softening effect was also blocked in the presence of SB-431542 (AngII + SB-431542 Z-groove $2.78 \pm 0.14 \mathrm{kPa}$, crest $3.06 \pm 0.16 \mathrm{kPa} v s$. control, $p>0.05$ ) (Fig. $1 \mathrm{~B}$ and D). Under these experimental conditions, the AngII effect on the transverse YM is caused by TGF- $\beta 1$ trans-activation. Recently, Ras/ Rho has been shown to be a part of the downstream signaling pathway of TGF- $\beta 1^{36}$ and that Rho is activated in neonatal cardiomyocytes following AngII treatment. ${ }^{37}$ We analyzed whether this pathway plays a role in the cell's YM using a selective Rho kinase inhibitor, Y-27632. Interestingly, AngII and Y-27632 together eliminate the decrease in YM seen after AngII treatment alone (AngII + Y-27632 Z-groove $4.13 \pm 0.38 \mathrm{kPa}$, crest $4.26 \pm 0.35 \mathrm{kPa}$, vs. AngII, $p<0.001$ ) (Fig. 1B and D) even observing an increase in the YM of these cells, although not statistically significant versus the control $(p>0.05)$.

\section{AngII targets microtubule post-translational modifications}

Published reports support the notion that Rho kinase targets both actin and the microtubular network; cell components known to increase cell YM with increased polymerization. Rho kinase activation results in actin polymerization, whereas Rho suppression results in a higher level of microtubule PTMs. ${ }^{38-40}$

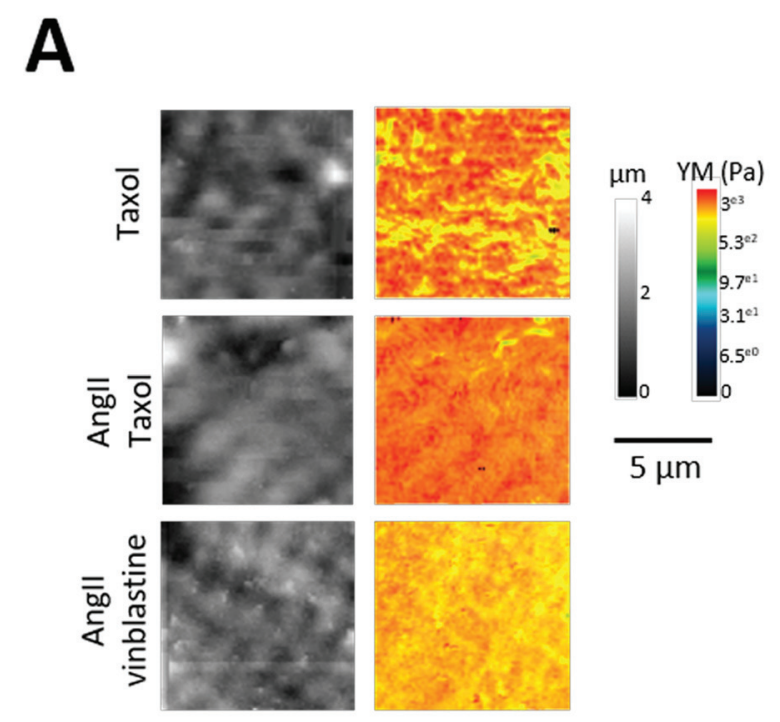

Elegant work from the Prosser group has shown that detyrosinated microtubules (MTs) contribute to cardiomyocyte mechanical properties ${ }^{8,41}$ and $\mathrm{Xu}$ et al., reported that acetylated microtubules modify cell mechanics. ${ }^{42,43}$ The AngII softening effect shown in this work cannot be explained by actin filaments playing the major role in YM regulation as activated Rho kinase would increase the cell's YM, which is not observed here. Therefore, we hypothesized that the microtubules and their PTMs are responsible for lowering the cell's YM in the short term, and this softening will be reversed in the long term by the increase in polymerization, which will result in cell stiffening.

To establish the involvement in MTs in cellular mechanics, we used the MT stabilizing agent taxol in order to promote tubule polymerization, and vinblastine that decreases the MT network as we have shown before. ${ }^{23}$ A significant increase in the cell's YM occurs following the treatment with AngII and taxol (AngII + taxol Z-groove $4.3 \pm 0.25 \mathrm{kPa}$; crest $4.45 \pm 0.24$ $\mathrm{kPa}$ vs. AngII; $p>0.01$ (Fig. 2A and B). On the other hand, AngII and vinblastine together show reduced YM values but did not show a cumulative effect (AngII + vinblastine Z-groove $1.96 \pm 0.18 \mathrm{kPa}$, crest $2.08 \pm 0.13 \mathrm{kPa} v$. AngII, $p>0.05)$ (Fig. 2A and B), suggesting a common signaling pathway.

MTs are formed by $\alpha$ and $\beta$-tubulin, where not only their amount is important, but also the stability which is regulated via PTMs. ${ }^{44}$ We quantified the total (polymerized and nonpolymerized) protein level of the main microtubule components: $\beta$-tubulin, $\alpha$-tubulin, and detyrosinated and acetylated $\alpha$-tubulins using western blot (Fig. 3A and B) under different pharmacological treatments. AngII treatment did not change the level of $\alpha$ - and $\beta$-tubulin. However, tubulin PTMs exhibit a trend towards the decreased level following AngII

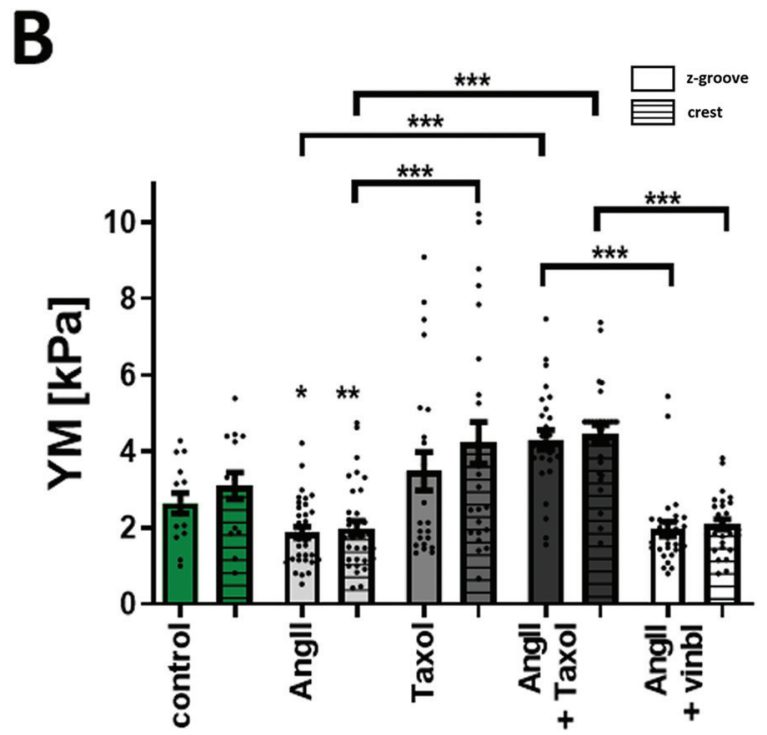

Fig. 2 Effect of microtubule modulation on YM. (A) Representative $10 \mu \mathrm{m} \times 10 \mu \mathrm{m}$ SICM images of topography and YM maps. (B) Graph summarizing the YM values obtained from the YM maps. Microtubule stabilization (taxol) significantly increases cellular YM. Simultaneous treatment of Angll with vinblastine does not further decrease YM when compared to Angll only treated cells. $N / n=3 / 15-35$. Data presented as \pm SEM. ${ }^{*} p<0.05$, ${ }^{* *} p<$ $0.01, * * * p<0.001$ 
treatment that could be potentially blocked by taxol or the TGF- $\beta 1$ inhibitor (Fig. 3A and B). Next, we performed immunofluorescence staining of cardiac myocytes for $\beta$-tubulin and tubulin PTMs to visualize the effect of AngII by confocal microscopy imaging. Similarly to the WB results, AngII treatment did not reduce $\beta$-tubulin immunostaining, but the decrease in tubulin PTM immunostaining is statistically significant after AngII treatment (Fig. 3C and D), and it can be blocked by taxol or the TGF- $\beta 1$ inhibitor.

\section{Acute AngII treatment also reduces transverse YM in myocardial infarction myocytes}

Cardiac myocytes undergo several complex structural and functional changes during myocardial infarction (MI). ${ }^{45}$ Currently, it is widely recognized that the cardiac mechanical properties are different in cardiac pathology when compared to healthy conditions. A good indicator of the failing heart is the increased YM. ${ }^{46,47}$ Here, we confirm that heart failure produces an increase in transversal YM of rat adult cardiomyocytes. We also observe that the difference between Z-grooves and crests is more pronounced. Interestingly, our results also show that treatment with AngII for 2 hours exerts the same softening effect on failing as on control cells. A significant decrease in the cell's YM is observed in cardiomyocytes isolated from a failing rat heart after AngII treatment (MI + AngII Z-groove $2.28 \pm 0.11 \mathrm{kPa}$, crest $2.63 \pm 0.14 \mathrm{kPa} v s$. MI Z-groove $4.87 \pm 0.45 \mathrm{kPa}$, crest $6.11 \pm 0.73 \mathrm{kPa}, p<0.05$ ) (Fig. $4 \mathrm{~A}$ and $\mathrm{B}$ ). Representing a $38 \%$ fall and "normalizing" the YM, reaching the control value. We also confirm that this corresponds to a reduction in the polymerized $\beta$-tubulin, based on the confocal micrograph analysis of immunostaining ( $\beta$-tubulin $\mathrm{MI}+$ AngII $0.45 \pm 0.02$ a.u. vs. MI $0.57 \pm 0.03$ a.u., $p<0.01$ ) (Fig. $4 \mathrm{C}$ and D), which was increased when compared to control cardiomyocytes. This confirms a beneficial effect of short-term treatment with AngII in the failing cardiomyocytes.

\section{Chronic AngII treatment evokes an opposite effect on cellular YM}

The canonical effect of chronic AngII treatment in the literature had been mostly related to hypertrophy and consequently an increase in YM. We confirmed that over 24-hour treatment AngII promotes cellular hypertrophy (AngII 24 h 248.1.6 \pm $10.5 \mu \mathrm{m}^{2}$ vs. control $24 \mathrm{~h} 215.6 \pm 8 \mu \mathrm{m}^{2}, p<0.05$ ) (Fig. 5A). The cellular YM did not decrease as with the short-term treatment, the cells exhibit a non-significant increase in YM when compared to non-treated 24-hour cells (24 h control Z-groove $2.32 \pm$ $0.24 \mathrm{kPa}$, crest $3.15 \pm 0.38 \mathrm{kPa}$ vs. $24 \mathrm{~h}$ AngII Z-groove $2.87 \pm$ $0.24 \mathrm{kPa}$, crest $3.6 \pm 0.35 \mathrm{kPa}, p>0.05$ ) (Fig. 5B and C). Based on immunofluorescence staining, we don't observe any change in $\beta$-tubulin or a decrease in the MT PTMs, but a small trend towards the increase in acMT (acMT $24 \mathrm{~h}$ AngII $0.24 \pm 0.01$ a.u. vs. $24 \mathrm{~h} 0.22 \pm 0.01$ a.u., $p>0.05$ ) and detyrMT (detyrMT $24 \mathrm{~h}$ AngII $0.23 \pm 0.01$ a.u. vs. 24 h $0.19 \pm 0.01$ a.u., $p>0.05)$ (Fig. 5D and E).

\section{Discussion}

The role of the mechanical properties in the regulation of the structural and functional properties of biological samples is now widely accepted due to the arrival of new techniques in the last 50 years. In this work, we present non-contact mechanoSICM that allows resolving the topography and the cell transverse YM of the same area simultaneously. Apart from the advantage that both live and fixed cells can be probed without a special preparation process, the system works in non-contact mode, so there is no concern regarding sample damage. By applying a topographical slope correction, we address the problem of underestimating the YM values in uneven and inclined sample surfaces. Another major advantage of this system is the high-resolution imaging that allows specific selection of membrane subdomains and examination of the YM in these areas. This technique selection mode opens the possibility to study nanoscale structures present at the membrane. We applied this tool to measure YM in adult cardiac myocytes known to form different membrane structures, such as Z-grooves and crests that express a number of varying proteins and channels in each that can contribute to different mechanical properties. Specifically, the cardiac myocyte sarcolemma is rich in mechanically gated channels that include stretch activated channels (SACs) and volume activated channels. Their main role is regulating myocardial contractile behaviour, but in the disease setting their function is defective. A wide number of effects caused by the faulty mechanosensitive channels in the chronic arrhythmias have been reported, such as an increase in the channel expression, action potential duration change caused by the channel activation, conduction velocity slowing, tissue remodelling and changes in the Connexin 43 phosphorylation level. ${ }^{48-53}$ Thus far, studies on the mechanosensitive channels were mostly underestimated, but the number of effects only in the heart shows that these structures require more thorough investigation. Notably, evidence demonstrating the presence of SACs has been found not only in cardiac myocytes, but also in a number of other cell types such as hair cells and epithelial cells. Also, mechanosensitive channels have been found in bacteria, archea, fungi and plants. Therefore, the presented technique could accelerate the structural and functional studies of membrane channels.

Generally, short-term treatment with AngII was found to make cells softer by affecting the transverse YM. Our results correlate with the data published by others ${ }^{13}$ that showed a decrease in longitudinal YM after AngII on rabbit papillary muscles. Although the AngII concentration chosen in this work is commonly used in in vitro models, the clinical utility of this concentration is questionable, since AngII in plasma concentrations from heart failure patients are in the picomolar range. ${ }^{5,55}$ Further studies are needed to understand whether the activation pathway described here could have a relevance in vivo. Studies in different cell models show that the increased YM of the extracellular matrix activates mechanosensing through integrin signaling. ${ }^{56,57}$ Moreover, a recent work by 

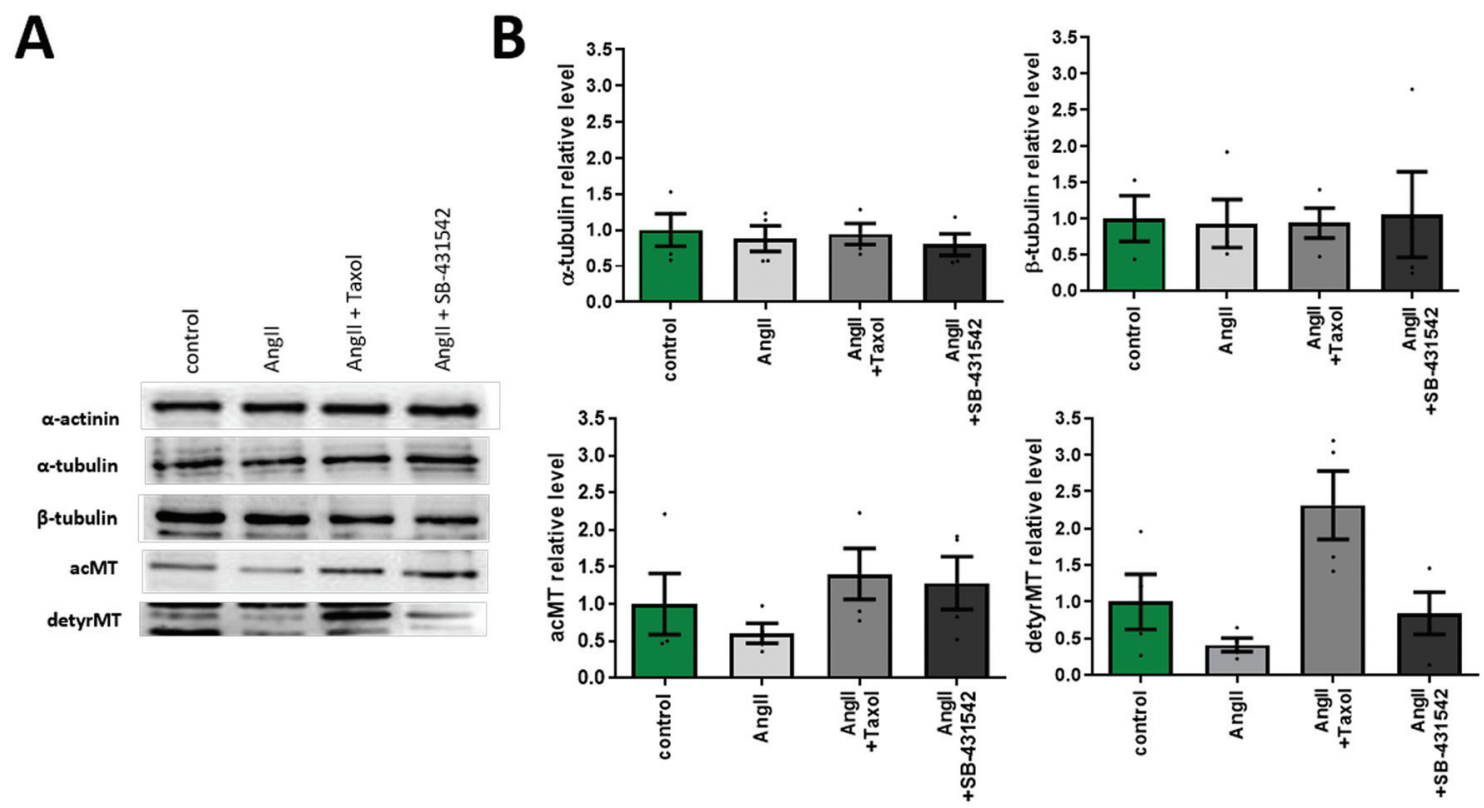

C

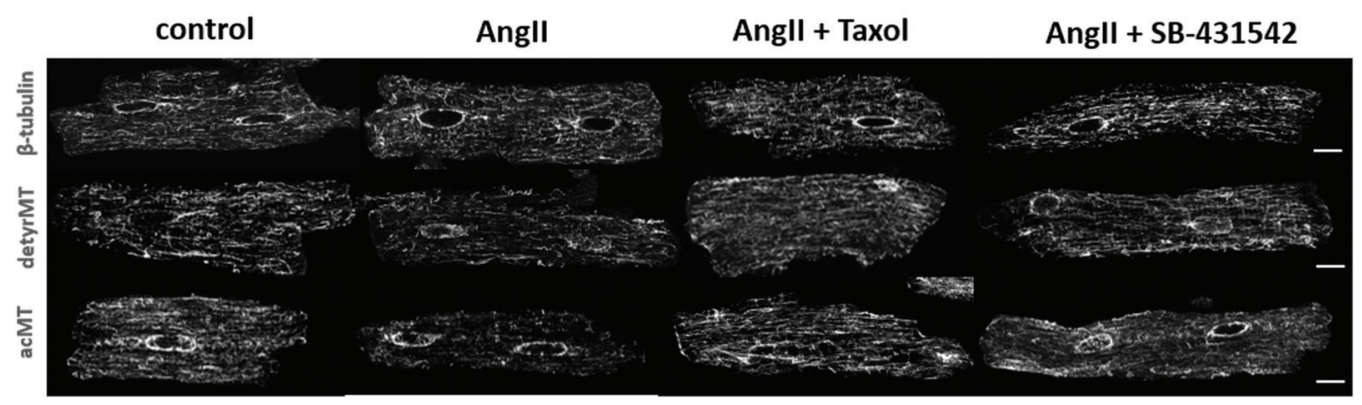

D
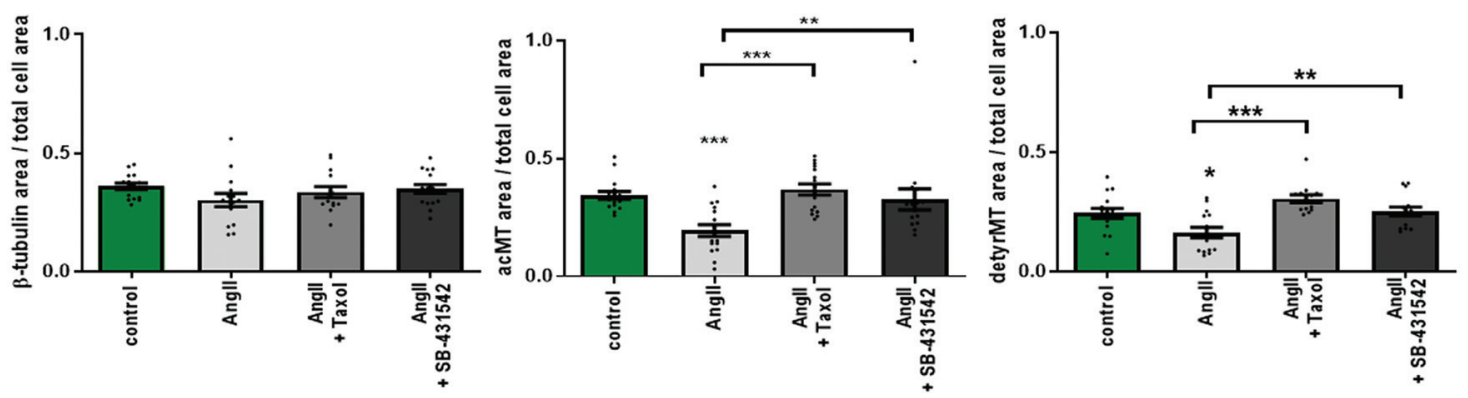

Fig. 3 Effect of Angll on $\alpha-$, $\beta$-tubulin, acetylated and detyrosinated microtubule populations. (A) Representative western blot used for densitometry quantification shown in (B). Summary graphs of WB analysis of $\alpha$-tubulin, $\beta$-tubulin, acetylated and detyrosinated microtubules with different treatments $N=4$. (C) Representative confocal micrographs. Cells stained for $\beta$-tubulin, acetylated and detyrosinated microtubules in four conditions, control, Angll, Angll + taxol, and Angll + SB-431542. Scale bar $10 \mu \mathrm{m}$. (D) Densitometry of fluorescence intensity of micrographs shown in (E). N/n $=$ 3/14-16. Data presented as mean \pm SEM. ${ }^{*} p<0.05,{ }^{* *} p<0.01,{ }^{* * *} p<0.001$.

Fiore et al. demonstrated that an increase in the cortical stiffness of fibroblasts positively correlates with the up-regulation of $\alpha v \beta 3$ integrin in focal adhesions. ${ }^{58}$ Further work is needed, to verify the correlation between $\mathrm{YM}$ and integrins, the potential of this pathway in diseases, and the relevance these studies may have in vivo.
Work by Chaturvedi et al. shows that a higher ventricle stiffness is correlated with an increased stiffness of both the extracellular matrix and isolated cardiomyocytes in the pressure but not volume-overload samples. ${ }^{59}$ These data confirm the need for studying the organization of different components and levels in the heart. In the Leite-Moreira 
A
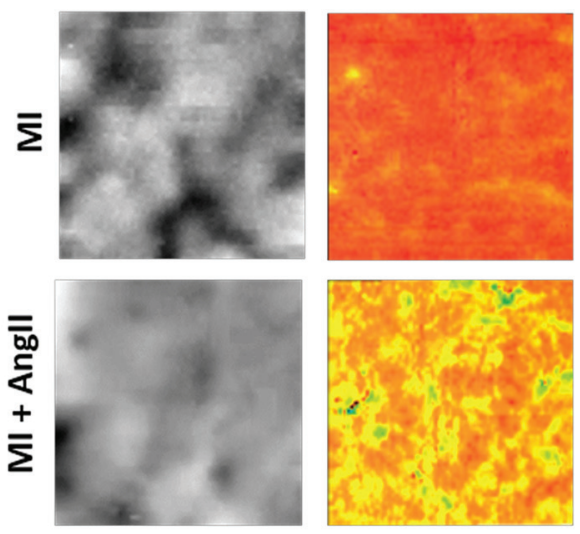

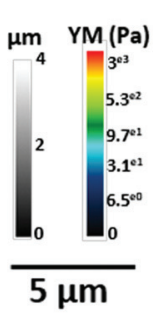

B
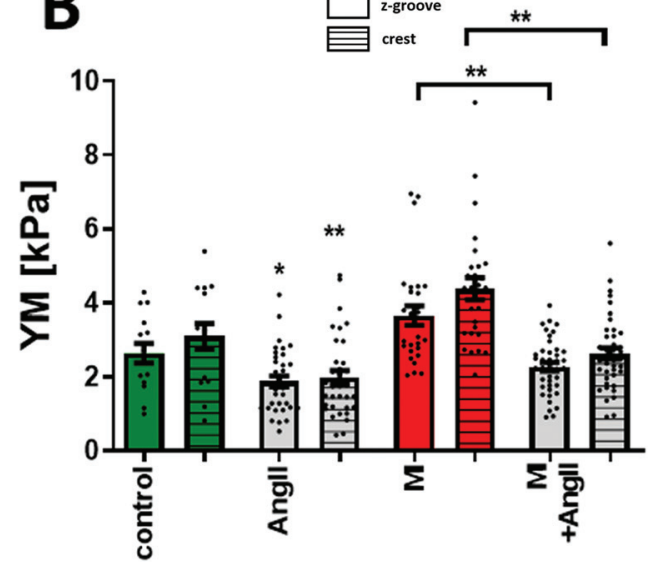

C

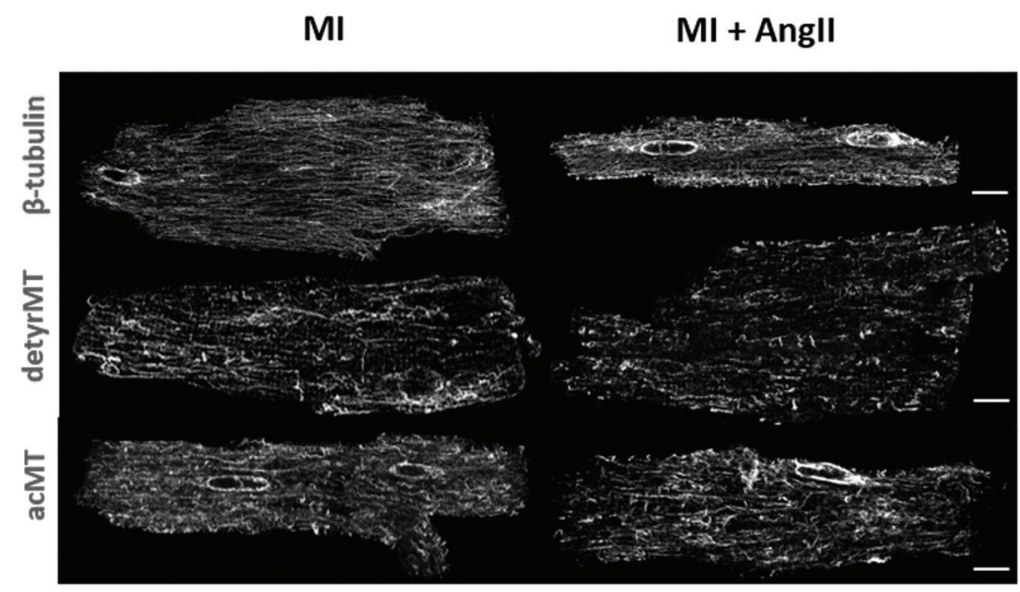

D
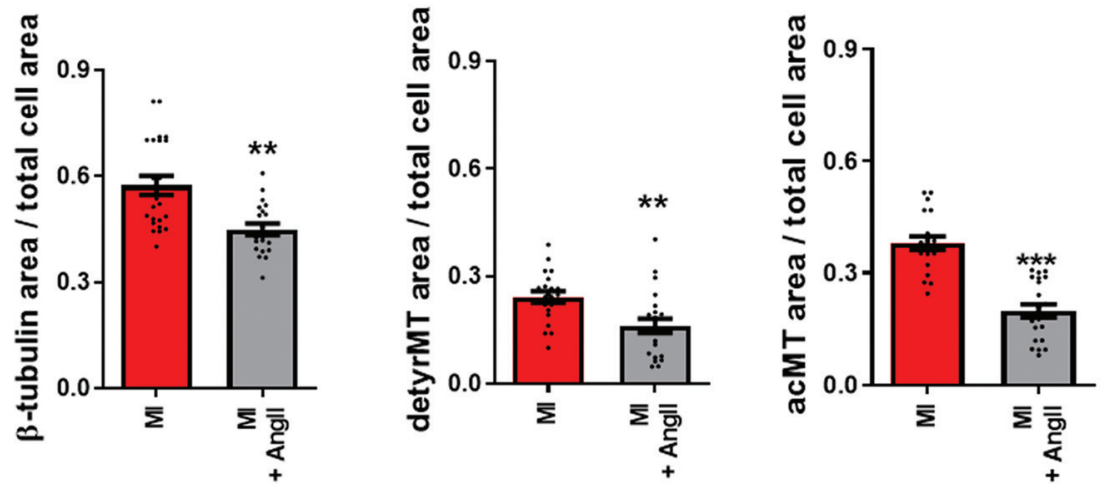

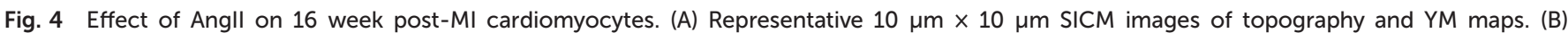

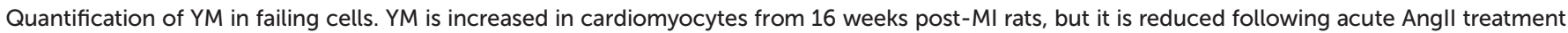

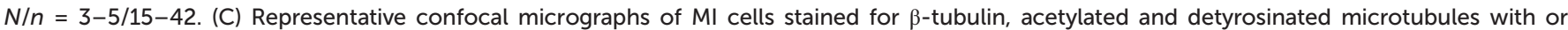

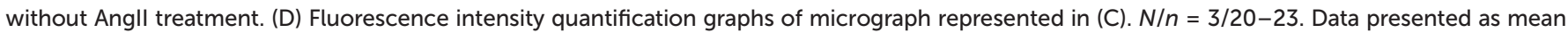
\pm SEM. ${ }^{* *} p<0.01 ;{ }^{* * *} p<0.001$.

study ${ }^{13}$ work by Chaturvedi et al., the mechanism of action wasn't fully revealed, however the changes in longitudinal passive-tension is likely to be primarily due to the modifications of titin, ${ }^{60}$ myofilaments ${ }^{61}$ and extracellular matrix pro- teins. ${ }^{59}$ Transverse YM, as measured in the present study, is a measure of membrane displacement and therefore it is likely to be modulated mostly by subsarcolemmal mitochondria, ${ }^{20}$ microtubules, ${ }^{23}$ and myofilaments. ${ }^{62}$ 
A

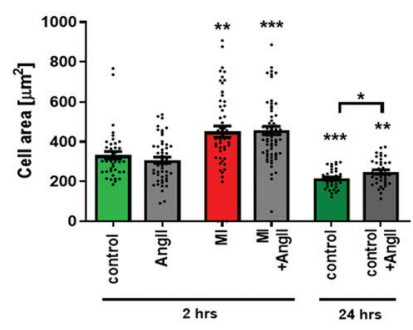

B
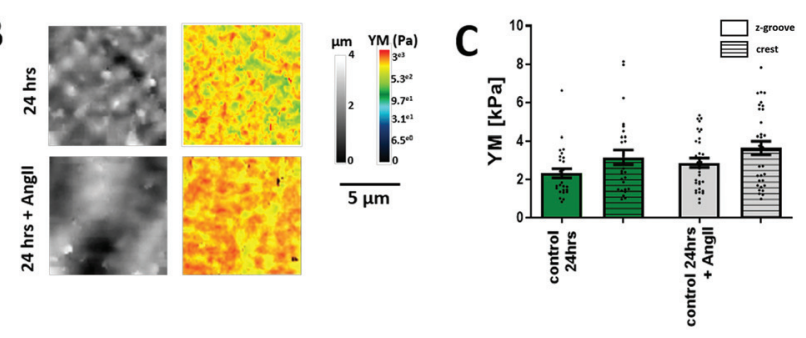

D

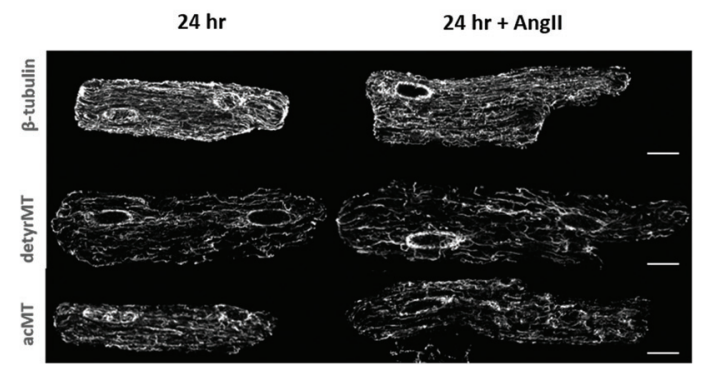

$\mathbf{E}$
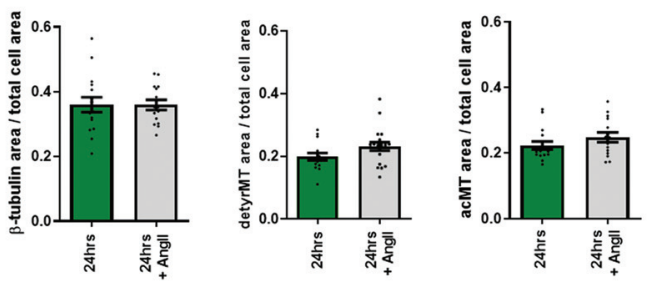

Fig. 5 Angll incubation for 24 hours exhibits an opposite effect on YM to short-term treatment for 2 hours. (A) Graph showing a cell area measured from confocal images. $N / n=5-6 / 41-64$. (B) Representative $10 \mu \mathrm{m} \times 10 \mu \mathrm{m}$ SICM images of topography and YM maps of myocytes incubated for 24 hours with or without Angll. (C) Quantification of YM, an increased cellular YM can be seen after $24 \mathrm{~h}$ Angll treatment. $\mathrm{N} / n=$ 3-4/27-33. (D) Representative confocal micrographs of 24 hours cultured cells with or without Angll treatment stained for $\beta$-tubulin, acetylated and detyrosinated microtubules. (E) Immunofluorescence intensity quantification graphs of cells from (C). $N / n=3 / 15-16$. Data presented as mean \pm SEM. ${ }^{*} p<0.05,{ }^{* *} p<0.01 ;{ }^{* * *} p<0.001$.

We show here that the activation of the specific AngII receptor AT1 is necessary to initiate this signaling cascade. Next, direct activation of the TGF- $\beta 1$ receptor is observed following the AngII-AT1R binding, similar to that seen in the renal epithelial cell hypertrophy. ${ }^{63}$ Our results further indicate that Rho kinase plays a significant role in the downstream of the TGF$\beta 1$ receptor, related to the MT density and PTMs. In fact, stabilizing MTs with taxol (Fig. 2) abolishes the softening effect of AngII exerted on cardiomyocytes. Previously, TGF- $\beta 1$ activation by AngII has been shown to be one of the crucial factors driving hypertrophy induced by AngII, affecting not only cardi- omyocytes but also fibroblasts. ${ }^{64}$ Our work extends the current understanding of cardiomyocyte remodeling, showing that AngII affects cellular transverse YM in the short term via TGF$\beta 1$ activation.

Recent studies performed on mouse aortic epithelial cells found that AngII caused MT network redistribution. ${ }^{65}$ MT stability is regulated by several PTMs; specifically, acetylation and detyrosination were shown to contribute to cellular mechanics. ${ }^{41,42}$ The detyrosinated population adopts a sinusoidal shape and increases its viscoelastic resistance, which is sensed by the myocyte sarcomere, due to the direct desmindependent connection. The acetylated MT pool provides protection from the damage during constant bending by providing a supportive framework. ${ }^{41,42}$ We see in our experiments that when cell transverse YM decreases as a result of AngII action, the amount of detyrosinated MTs also reduces, as does the amount of acetylated MTs (Fig. 3). How AngII regulates tubulin modifications is unclear at present, although a possible explanation is that AngII acts through a Rho-kinase pathway.

The Rho family of small G proteins is known to regulate cellular dynamics. ROCK is shown to act on the actin cytoskeleton through the phosphorylation of the myosin light chain, LIM kinase and MLC phosphatase as the main targets. ${ }^{66}$ Further research showed that the Rho family can also affect microtubule dynamics. ${ }^{67,68}$ In fact, the Rho kinase-microtubule relation is not linear; a reciprocal interaction had been described where microtubules can also control the G protein. Blocking the Rho kinase with a specific inhibitor increases acetylated and detyrosinated tubulin. ${ }^{69-71}$ This has been shown to be due to the tubulin polymerization promoting the interaction of protein 1 and histone deacetylase 6 , which eventually prevents microtubule deacetylation. ${ }^{38}$ In our study, we see that an AngII-dependent decrease in cell transverse YM is abolished after inhibiting Rho kinase (Fig. 1B and D). We propose that by inhibiting Rho using Y27632, the detyrosinated MT pool is increased, stiffening the cells, and AngII cannot effectively reduce the transverse YM after this, since the Rho signaling has been blocked, suggesting that AngII may affect the microtubular network at least in part through Rho activation. In this case, the inhibition of Rho that will also increase the acetylated MTs and, theoretically, causing softening of the cells, but this effect is overpowered by the detyrosinated MT effect, as it has been suggested before (Swiatlowska et al., 2020). ${ }^{23}$ Although, we cannot exclude that there are other mechanisms such as ERK 1/2 activation, we and others have shown before that failing myocytes are stiffer than healthy myocytes and that they express higher levels of microtubules as a result of a pathological insult..$^{8,20,23}$ We tested whether the short-term softening effect of AngII is present in cells from the rat model of MI. Surprisingly, despite undergoing extensive remodeling after MI, failing cells demonstrate a decrease in transverse YM by 38\% following treatment with AngII accompanied by a reduction in MTs (Fig. 4). It has been reported that MT depolymerization normalizes cell contractility in the hypertrophied myocardium. ${ }^{72}$ In human failing 
hearts, inhibition of detyrosinated MTs by a genetic or pharmacological approach brings functional improvement, ${ }^{8}$ which included an improved contractility function as well as a reduction in cardiomyocyte $\mathrm{YM}^{41}$ In our experiments, AngIItreated failing cells demonstrate a reduction in the amount of $\beta$-tubulin as well as detyrosinated and acetylated MTs, compared with non-treated failing cells, to levels comparable with control values.

\section{Conclusion}

To summarize, this work describes a transition period under AngII stimulation, showing a shift from the adaptive (shortterm) to maladaptive (long-term) state, and the importance of the AngII/TGF-B1 pathway that leads to microtubule remodeling through the involvement of the Rho kinase pathway. Also, mechanoSICM has been shown to be a well-suited tool for studying the membrane mechanical properties in living cells, and it can precisely resolve areas of different YM values on the surface of cells which may correspond to signaling microdomains, as shown for Z-grooves and crests, found in cardiac myocytes.

\section{Materials and methods}

\section{Cell isolation and culture}

All animal experiments were carried out in accordance with the United Kingdom Home Office Animals (Scientific Procedures) Act 1986 Amendment Regulations 2012, incorporating the EU Directive 2010/63/EU. Approval for this work was obtained from the Animal Welfare and Ethics Review Board (AWERB) of Imperial College London. Adult Sprague-Dawley rats were used as controls and the myocardial infarction (MI) model was generated by ligation of the left anterior descending coronary artery; all the characteristics of MI develop after 16 weeks, as mentioned in detail previously. ${ }^{45,73}$

Adult rat cardiomyocyte isolation was performed as previously reported. ${ }^{45}$ Briefly, Langendorff perfusion was used to isolate left ventricle cardiomyocytes from male adult SpragueDawley rats weighing 150-250 g. Hearts were extracted and Langendorff-perfused with first tyrode solution (5 minutes), second with low $\mathrm{Ca}^{2+}$ solution (5 minutes), and finally with enzyme solution containing collagenase $\left(1 \mathrm{mg} \mathrm{ml}^{-1}\right.$; Worthington) and hyaluronidase (0.6 $\mathrm{mg} \mathrm{ml}^{-1}$; Sigma-Aldrich) for 10 minutes. Afterwards, hearts were removed from the perfusion system and the left ventricle was removed, cut into smaller pieces, and re-suspended in enzyme solution but containing only collagenase, and shaken for 30 minutes. Finally, cell suspension was centrifuged and left in buffer solution without enzymes at room temperature.

The cells were cultured in M199 media (Gibco) supplemented with taurine $5 \mathrm{mM}$ (Sigma), carnitine $5 \mathrm{mM}$ (Sigma), creatine $5 \mathrm{mM}$ (Sigma), $0.002 \mathrm{~g} \mathrm{ml}^{-1}$ bovine serum albumin (Sigma), ascorbate $100 \mathrm{mM}$ (Sigma) and $100 \mathrm{mM}$ penicillin/streptomycin.

The following times and concentrations of reagents were used for treatments at $37{ }^{\circ} \mathrm{C}$ : Angiotensin II (Tocris) $20 \mu \mathrm{M}$, 2 hours; Losartan (Abcam) $100 \mu \mathrm{M}, 30$ minutes; 5-( $N$-methyl- $N$ isobutyl)-amiloride (MIA) $\mathrm{Na}^{+} / \mathrm{H}^{+}$inhibitor (Tocris) $1 \mu \mathrm{M}$, 2.5 hours; Vinblastine (Sigma) $2 \mu \mathrm{M}, 1$ hour; Y-27632 (Sigma) $5 \mu \mathrm{M}, 1$ hour; SB-431542 (Tocris), $10 \mu \mathrm{M}, 2$ hours; Taxol (Sigma) $10 \mu \mathrm{M}, 3$ hours.

\section{A MechanoSICM setup}

A Scanning Ion Conductance Microscope was used to generate topography images. Further system modifications (mechanoSICM) were applied to generate Young's modulus maps.

Tubing attached to the pressure side port of the nanopipette holder was used to manually deliver aerostatic pressure that propels the inner pipette solution forming a hydrojet. A positive pressure of $15 \mathrm{kPa}$ was applied constantly while scanning and monitored using a pressure meter (PM1000, World Precision Instruments). The inner diameter of the pipette determines the resolution of imaging. To generate a highresolution image, standard SICM pipettes typically have a $100 \mathrm{~nm}$ inner diameter ( $\sim 100 \mathrm{M} \Omega$ resistance) or less, but for the mechanical measurements, wider pipettes $(200 \mathrm{~nm}$ diameter and more) are preferred ${ }^{21}$ due to the lower risk of blockage when internal pressure is applied. In this work, we used pipettes with diameters in the range of 250-350 nm, which gives the best balance between the reliability of pressure application and the image resolution. Displacement of the membrane due to the pressure of pipette solution is registered by the nanopipette approaching the membrane at each imaging point. Scanning solution containing $144 \mathrm{mM} \mathrm{NaCl}, 10 \mathrm{mM}$ HEPES, $1 \mathrm{mM} \mathrm{MgCl}_{2}$, and $5 \mathrm{mM} \mathrm{KCl,} 7.4 \mathrm{pH}$ was used both as the pipette and bath solution.

\section{Measurements of elastic modulus}

For topography imaging in the hopping mode, ${ }^{25}$ the nanopipette approaches the sample surface until the current is reduced by a predefined amount (typical setpoint $\mathrm{SP}_{\mathrm{T}}=0.7 \%$ ). The position of the $z$-axis piezo-actuator at the moment the current achieves this reduction is recorded as the height of the sample at this imaging point and shown in the topography. For measurements of YM using the method first described by Rheinlaender and Schaffer, ${ }^{21}$ a positive pressure $p_{0}$ is applied to the pipette during imaging. The pipette travels further down after reaching the topographical setpoint $\mathrm{SP}_{\mathrm{T}}$ until the current is reduced by further $1 \%$ (to setpoint $\mathrm{SP}_{1}=1 \%$ ) while recording the distance travelled as $d_{1}$, and then even further down to $\mathrm{SP}_{2}=2 \%\left(2 \%\right.$ from $\left.\mathrm{SP}_{\mathrm{T}}\right)$ recording the distance travelled from $\mathrm{SP}_{\mathrm{T}}$ as $d_{2}$. This is repeated for all imaging points so that three images are recorded simultaneously. Rheinlaender and Schaffer showed that the current-distance curve between the setpoints $\mathrm{SP}_{1}$ and $\mathrm{SP}_{2}$ can be approximated by a straight line with a slope reflecting the Young's modulus $E$ of the sample 
surface. Here, we used a simplified calculation for membrane displacement.

In general, the stiffer surface will result in a steeper slope of the current-distance curve than the soft surface. The currentdistance relationship can be mathematically described as:

$$
E=p_{0} A\left(\frac{s_{\mathrm{R}}}{s}-1\right)^{-1}
$$

where $s_{\mathrm{R}}$ is the slope of the current-distance curve between $1 \%$ and $2 \%$ setpoints measured at an infinitely stiff surface (in practice, a cell culture dish appears stiff enough) representing the reference, and $s$ is the slope of the current-distance curve measured when approaching the cell surface (or other reasonably soft material), $p_{0}$ is the pressure applied to the nanopipette interior, and finally, $A$ is a constant characterising the geometry of the nanopipette.

In our implementation of the Rheinlaender and Schaffer method, the slope is calculated simply as:

$$
s=\frac{1}{d_{2}-d_{1}}\left[\% \mathrm{~nm}^{-1}\right]
$$

where $d_{2}$ and $d_{1}$ are the displacements at setpoints $\mathrm{SP}_{2}$ and $\mathrm{SP}_{1}$. Eqn (1) can then be rewritten into the following form:

$$
E=\frac{p_{0} A}{\frac{d_{2}-d_{1}}{d_{2 \mathrm{R}}-d_{1 \mathrm{R}}}-1}
$$

where $d_{2 \mathrm{R}}$ and $d_{1 \mathrm{R}}$ are the displacements measured on a stiff reference surface such as glass or PVC plastic of the tissue culture dish.

In practice, $d_{2 \mathrm{R}}$ and $d_{1 \mathrm{R}}$ displacements can be taken from a portion of the scan area not covered by cells, where the stiff substrate is exposed or from a separate image recorded on a stiff substrate using the same nanopipette.

YM is calculated after two setpoint images were obtained using the SICMImageViewer software (Chen et al. 2018b). ${ }^{74}$ The software uses the displacement data from images and eqn (3) to calculate YM of every imaged point of the sample surface. In this work, we have implemented additional correction into the SICMImageViewer in order to correct the effect of the local slope $\alpha$ estimated from the undeformed topography image obtained at a low setpoint as illustrated in ESI Fig. $1: \dagger$

$$
E=\frac{p_{0} A}{\frac{\left(d_{2}-d_{1}\right) \cos \alpha}{d_{2 \mathrm{R}}-d_{1 \mathrm{R}}}-1}
$$

The YM maps are measured simultaneously with topography images and have the same image resolution (effective pixel size), which was typically $20 \mathrm{~nm}$ for a $10 \mu \mathrm{m} \times 10 \mu \mathrm{m}$ scan. This allowed us to correlate the softer and stiffer areas with the corresponding cell membrane nanostructure. The invaginations in the topographical scans are the Z-grooves containing the T-tubule openings as the deepest invaginations, and the elevated areas between them are defined as the crests. For each topographical map, 6 regions of interest (ROI) of similar sizes were selected, 3 in the Z-groove (TT) area, and 3 in the Crest crest area, and the YM was calculated from the corresponding ROI on the YM map by the software. Only the center of the cell was probed avoiding the edges. All elastic modulus measurements were performed at room temperature.

\section{Immunofluorescence staining}

The cells were fixed in ice cold methanol for 5 minutes or $4 \%$ paraformaldehyde for 10 minutes, permeabilized in Triton X-100 for 15 minutes and blocked for 1 hour in 10\% horse serum or $1 \%$ Bovine Serum Albumin as a blocking buffer. Next, the cells were incubated with a primary antibody for 2 hours at RT, washed with PBS and incubated for another hour with a secondary antibody and Hoechst.

Primary antibodies were: anti-mouse $\beta$-tubulin (clone TU27, Cat No 903401; BioLegend), diluted 1 : 1000; anti-mouse acetylated tubulin (clone 6-11B-1, sc-23950; Santa Cruz Biotech), diluted 1:100; anti-rabbit detyrosinated tubulin (AB3201; Millipore), diluted 1:100. Secondary antibodies were all diluted 1:500: Alexa Fluor 488 donkey anti-mouse (A21202; ThermoFisher), Alexa Fluor 488 goat anti-rabbit (A11008, ThermoFisher); and Hoechst 33342 (V35118; Life Technologies) was used to stain nuclei. Coverslips were mounted using Prolong Gold (P10144, ThermoFisher). A confocal laser-scanning microscope (Zeiss LSM-780 inverted) with a Plan Apochromat $63 \times / 1.4$ oil immersion objective at room temperature was used. Data were acquired with the provided ZEN software (Zeiss). A $Z$-stack of images was taken from the top to the bottom of the cell in $1 \mu \mathrm{m}$ steps. For each cell, three images from the $z$-stack, from the cell centre, were averaged using maximum intensity projection, standardized to the area of respective cell size and quantified by default intensity thresholding using ImageJ (https://imagej.nih.gov/ij/).

\section{Western blotting}

The cells were washed once with cold PBS and lysed with RIPA buffer (R0278; Sigma) containing a protease inhibitor (P2714; Sigma). The samples were then sonicated for $1 \mathrm{~min}$, at $4{ }^{\circ} \mathrm{C}$ and left for $15 \mathrm{~min}$ gentle agitation. Lastly, the cells were spun down and the supernatant was kept for further use. The protein concentration was determined using a Pierce BCA Protein Assay Kit (\#23227F, Thermo Scientific). Proteins were separated by SDS-PAGE and then transferred onto a PVDF membrane and blocked for 1 hour with 5\% skimmed milk. Membranes were incubated with primary antibodies overnight at $4{ }^{\circ} \mathrm{C}$, rinsed 3 times with tris-buffered saline plus $0.5 \%$ Tween 20 (TBST) and probed with the secondary antibody for 1 hour, at RT followed by three TBST washes. An ECL Western Blotting Detection Reagent (GE Healthcare) was used to develop the membranes. Original WBs are shown in ESI Fig. $3 . \dagger$

Antibodies used for western blotting: anti-mouse $\alpha$-tubulin (clone TU02, sc-8035; Santa Cruz Biotech) diluted 1:250; anti$\beta$-tubulin, anti-acetylated tubulin and anti-detyrosinated tubulin. Secondary antibodies were used at 1:1000 dilution: anti-mouse HRP-linked (7076S, Cell Signalling) and anti-rabbit HRP-linked (7074S, Cell Signalling). Band optical density was 
measured using the Image software on images taken on Syngene G: BOX (Singene).

\section{Statistics}

Data analysis was performed using GraphPad Prism 5. Normality of the distribution was assessed by the KolmogorovSmirnov test. Next, Student's $t$ test or one-way analysis of variance (ANOVA) was used for statistical analysis. The number of examined samples is presented as $N / n$, where $N$ stands for the number of animal isolations, and $n$ represents the total number of analyzed cells. All graphs were depicted as standard error of the mean (SEM). $P$-value significance accepted as 0.05 $\left(^{*}\right), 0.01\left(^{* *}\right)$ and $0.001(* * *)$.

\section{Nonstandard abbreviations and acronyms}

$\begin{array}{ll}\text { AngII } & \text { Angiotensin II } \\ \text { AFM } & \text { Atomic force microscopy } \\ \text { PKC } & \text { Protein kinase C } \\ \text { AT1 } & \text { Angiotensin type } 1 \text { receptor } \\ \text { MTs } & \text { Microtubules } \\ \text { PTMs } & \text { Post-translational modifications } \\ \text { SICM } & \text { Scanning ion conductance microscopy } \\ \text { YM } & \text { Young's modulus } \\ \text { TGF- } \beta 1 & \text { Transforming growth factor- } \beta 1 \\ \text { MI } & \text { Myocardial infarction } \\ \text { TT } & \text { Transverse tubule } \\ \text { ERK } & \text { Extracellular signal-regulated kinase }\end{array}$

\section{Author contributions}

P. S. and J. G. designed the experiments, P. S. and D. S. performed the experiments, C. M. generated the animal model, P. N. provided the help with analysis of data, P. S. and J. L. S-A. wrote the manuscript, P. N., J. L. S-A., C. M., Y. K. and J. G. corrected the manuscript, and P. N., J. L. S-A., and J. G., provided supervision.

\section{Conflicts of interest}

The authors declare no competing interests.

\section{Acknowledgements}

We are most grateful to P. O'Gara for the isolation of adult rat ventricular cardiomyocytes. We thank B. Reilly-O'Donnell for manuscript check-up, V. Braga for providing the Y-compound reagent, and S. Rothery for confocal microscopy assistance. We would also like to thank B.L. Prosser and D. Kass for experimental suggestions and helpful discussion. This work was supported by British Heart Foundation (grant RG/17/13/33173 to J.
$G$ and J. S.) and Imperial College London NHLI PhD studentship. P. N. acknowledges the support from the Russian Science Foundation (grant no. 19-79-30062), Y. K. acknowledges the support from the World Premier International Research Center Initiative (WPI), MEXT, Japan.

\section{References}

1 S. K. Atkins, A. McNally and P. Sucosky, Mechanobiology in Cardiovascular Disease Management: Potential Strategies and Current Needs, Front. Bioeng. Biotechnol., 2016, 4, 79.

2 K. Takahashi, Y. Kakimoto, K. Toda and K. Naruse, Mechanobiology in cardiac physiology and diseases, J. Cell. Mol. Med., 2013, 17(2), 225-232.

3 L. Andrés-Delgado and N. Mercader, Interplay between cardiac function and heart development, Biochim. Biophys. Acta, Mol. Cell Res., 2016, 1863(7), 1707-1716.

4 M. S. Barnabei and J. M. Metzger, Ex Vivo Stretch Reveals Altered Mechanical Properties of Isolated DystrophinDeficient Hearts, PLoS One, 2012, 7(3), e32880.

5 J. G. Jacot, J. C. Martin and D. L. Hunt, Mechanobiology of cardiomyocyte development, J. Biomech., 2010, 43(1), 9398.

6 A. J. S. Ribeiro, Y.-S. Ang, J.-D. Fu, R. N. Rivas, T. M. A. Mohamed, G. C. Higgs, et al. Contractility of single cardiomyocytes differentiated from pluripotent stem cells depends on physiological shape and substrate stiffness, Proc. Natl. Acad. Sci. U. S. A., 2015, 112(41), 12705-12710.

7 M. A. Caporizzo, C. Y. Chen, K. Bedi, K. B. Margulies and B. L. Prosser, Microtubules Increase Diastolic Stiffness in Failing Human Cardiomyocytes and Myocardium, Circulation, 2020, 141(11), 902-915.

8 C. Y. Chen, M. A. Caporizzo, K. Bedi, A. Vite, A. I. Bogush, P. Robison, et al. Suppression of detyrosinated microtubules improves cardiomyocyte function in human heart failure, Nat. Med., 2018, 24(8), 1225-1233.

9 H. F. J. Dullens, M. E. I. Schipper, J. Van Kuik, W. Sohns, M. Scheenstra, D. F. Van Wichen, et al. Integrin expression during reverse remodeling in the myocardium of heart failure patients, Cardiovasc. Pathol., 2012, 21(4), 291-298.

10 P. T. Wright, J. L. Sanchez-Alonso, C. Lucarelli, A. AlvarezLaviada, S. Bello, G. Faggian, et al. Partial mechanical unloading of the heart disrupts L-type calcium channel and beta-adrenoceptor signalling microdomains, Front. Physiol., 2018, 9, 1302.

11 M. A. Rossi and S. V. Carillo, Cardiac hypertrophy due to pressure and volume overload: distinctly different biological phenomena?, Int. J. Cardiol., 1991, 31(2), 133-141.

12 L. T. Skeggs, F. E. Dorer, J. R. Kahn, K. E. Lentz and M. Levine, The biochemistry of the renin-angiotensin system and its role in hypertension, Am. J. Med., 1976, 60(6), 737-748.

13 A. F. Leite-Moreira, P. Castro-Chaves, P. Pimentel-Nunes, A. Lima-Carneiro, M. S. Guerra, J. B. Soares, et al. Angiotensin II acutely decreases myocardial stiffness: a 
novel AT1, PKC and Na, Br. J. Pharmacol., 2006, 147, 690697.

14 M. Khalilian, M. Navidbakhsh, M. R. Valojerdi, M. Chizari and P. E. Yazdi, Estimating Young's modulus of zona pellucida by micropipette aspiration in combination with theoretical models of ovum, J. R. Soc., Interface, 2010, 7(45), 687-694.

15 R. M. Hochmuth, Micropipette aspiration of living cells, J. Biomech., 2000, 33(1), 15-22.

$16 \mathrm{H}$. Zhang and K.-K. Liu, Optical tweezers for single cells, J. R. Soc., Interface, 2008, 5(24), 671-690.

17 Y. A. Ayala, B. Pontes, D. S. Ether, L. B. Pires, G. R. Araujo, S. Frases, et al. Rheological properties of cells measured by optical tweezers, BMC Biophys., 2016, 9, 5.

18 E. Dague, G. Genet, V. Lachaize, C. Guilbeau-Frugier, J. Fauconnier, C. Mias, et al. Atomic force and electron microscopic-based study of sarcolemmal surface of living cardiomyocytes unveils unexpected mitochondrial shift in heart failure, J. Mol. Cell. Cardiol., 2014, 74, 162-172.

19 S. L. Crick and F. C.-P. Yin, Assessing Micromechanical Properties of Cells with Atomic Force Microscopy: Importance of the Contact Point, Biomech. Model. Mechanobiol., 2007, 6(3), 199-210.

20 M. Miragoli, J. L. Sanchez-Alonso, A. Bhargava, P. T. Wright, M. Sikkel, S. Schobesberger, et al. Microtubule-Dependent Mitochondria Alignment Regulates Calcium Release in Response to Nanomechanical Stimulus in Heart Myocytes, Cell Rep., 2016, 14(1), 140-151.

21 J. Rheinlaender and T. E. Schäffer, Mapping the mechanical stiffness of live cells with the scanning ion conductance microscope, Soft Matter, 2013, 9(12), 3230.

22 J. Rheinlaender and T. E. Schäffer, Mapping the creep compliance of living cells with scanning ion conductance microscopy reveals a subcellular correlation between stiffness and fluidity, Nanoscale, 2019, 11(14), 69826989.

23 P. Swiatlowska, J. L. Sanchez-Alonso, P. T. Wright, P. Novak and J. Gorelik, Microtubules regulate cardiomyocyte transversal Young's modulus, Proc. Natl. Acad. Sci. U. S. A., 2020, 117(6), 201917171.

24 Y. E. Korchev, C. L. Bashford, M. Milovanovic, I. Vodyanoy and M. J. Lab, Scanning ion conductance microscopy of living cells, Biophys. J., 1997, 73(2), 653-658.

25 P. Novak, C. Li, A. I. Shevchuk, R. Stepanyan, M. Caldwell, S. Hughes, et al. Nanoscale live-cell imaging using hopping probe ion conductance microscopy, Nat. Methods, 2009, 6(4), 279-281.

26 D. Thatenhorst, J. Rheinlaender, T. E. Schäffer, I. D. Dietzel and P. Happel, Effect of Sample Slope on Image Formation in Scanning Ion Conductance Microscopy, Anal. Chem., 2014, 86(19), 9838-9845.

27 S. A. Griffin, W. C. B. Brown, F. MacPherson, J. C. McGrath, V. G. Wilson, N. Korsgaard, et al. Angiotensin II causes vascular hypertrophy in part by a non-pressor mechanism, Hypertension, 1991, 17(5), 626-635.
28 T. W. Emans, D. Patinha, J. A. Joles, M. P. Koeners, B. J. Janssen and C. T. P. Krediet, Angiotensin II-induced hypertension in rats is only transiently accompanied by lower renal oxygenation, Sci. Rep., 2018, 8(1), 1-9.

29 M. Zhang, B. L. Prosser, M. A. Bamboye, A. N. S. Gondim, C. X. Santos, D. Martin, et al. Contractile Function during Angiotensin-II Activation: Increased Nox2 Activity Modulates Cardiac Calcium Handling via Phospholamban Phosphorylation, J. Am. Coll. Cardiol., 2015, 66(3), 261-272.

30 H. Duan, Y. Li, L. Yan, H. Yang, J. Wu, P. Qian, et al. Rcan1$1 \mathrm{~L}$ overexpression induces mitochondrial autophagy and improves cell survival in angiotensin II-exposed cardiomyocytes, Exp. Cell Res., 2015, 335(1), 99-106.

31 H. Ito, Y. Hirata, S. Adachi, M. Tanaka, M. Tsujino, A. Koike, et al. Endothelin-1 is an autocrine/paracrine factor in the mechanism of angiotensin II-induced hypertrophy in cultured rat cardiomyocytes, J. Clin. Invest., 1993, 92(1), 398-403.

32 F. Perbellini, S. A. Watson, M. Scigliano, S. Alayoubi, S. Tkach, I. Bardi, et al. Investigation of cardiac fibroblasts using myocardial slices, Cardiovasc. Res., 2018, 114(1), 7789.

33 C. Kupfahl, D. Pink, K. Friedrich, H. Zurbrügg, M. Neuss, C. Warnecke, et al. Angiotensin II directly increases transforming growth factor beta1 and osteopontin and indirectly affects collagen mRNA expression in the human heart, Cardiovasc. Res., 2000, 46(3), 463-475.

34 J. Sadoshima and S. Izumo, Molecular characterization of angiotensin II-induced hypertrophy of cardiac myocytes and hyperplasia of cardiac fibroblasts. Critical role of the AT1 receptor subtype, Circ. Res., 1993, 73(3), 413-423.

35 S. Wenzel, G. Taimor, H. M. Piper and K. D. Schluter, Redox-sensitive intermediates mediate angiotensin IIinduced p38 MAP kinase activation, AP-1 binding activity, and TGF- $\beta$ expression in adult ventricular cardiomyocytes, FASEB J., 2001, 15(12), 2291-2293.

36 E. Nomikou, M. Livitsanou, C. Stournaras and D. Kardassis, Transcriptional and post-transcriptional regulation of the genes encoding the small GTPases RhoA, RhoB, and RhoC: implications for the pathogenesis of human diseases, Cell. Mol. Life Sci., 2018, 75(12), 21112124.

37 M. Nishida, S. Tanabe, Y. Maruyama, S. Mangmool, K. Urayama, Y. Nagamatsu, et al. G alpha 12/13- and reactive oxygen species-dependent activation of c-Jun NH2terminal kinase and p38 mitogen-activated protein kinase by angiotensin receptor stimulation in rat neonatal cardiomyocytes, J. Biol. Chem., 2005, 280(18), 18434-18441.

38 A. V. Schofield, R. Steel and O. Bernard, Rho-associated Coiled-coil Kinase (ROCK) Protein Controls Microtubule Dynamics in a Novel Signaling Pathway That Regulates Cell Migration, J. Biol. Chem., 2012, 287(52), 4362043629.

39 D. E. Ingber, D. Prusty, Z. Sun, H. Betensky and N. Wang, Cell shape, cytoskeletal mechanics, and cell cycle control in angiogenesis, J. Biomech., 1995, 28(12), 1471-1484. 
40 S. Sharma, C. Santiskulvong, J. Rao, J. K. Gimzewski and O. Dorigo, The role of Rho GTPase in cell stiffness and cisplatin resistance in ovarian cancer cells, Integr. Biol., 2014, 6(6), 611-617.

41 P. Robison, M. A. Caporizzo, H. Ahmadzadeh, A. I. Bogush, C. Chen, K. B. Margulies, et al. Detyrosinated microtubules buckle and bear load in contracting cardiomyocytes, Science, 2016, 352(6284), aaf0659.

42 Z. Xu, L. Schaedel, D. Portran, A. Aguilar, J. Gaillard, M. P. Marinkovich, et al. Microtubules acquire resistance from mechanical breakage through intralumenal acetylation, Science, 2017, 356(6335), 328-332.

43 D. Portran, L. Schaedel, Z. Xu, M. Théry and M. V. Nachury, Tubulin acetylation protects long-lived microtubules against mechanical ageing, Nat. Cell Biol., 2017, 19(4), 391398.

44 Y. Song and S. T. Brady, Post-translational modifications of tubulin: pathways to functional diversity of microtubules, Trends Cell Biol., 2015, 25(3), 125-136.

45 A. R. Lyon, K. T. MacLeod, Y. Zhang, E. Garcia, G. K. Kanda, M. J. Lab, et al. Loss of T-tubules and other changes to surface topography in ventricular myocytes from failing human and rat heart, Proc. Natl. Acad. Sci. U. S. A., 2009, 106(16), 6854-6859.

46 L. van Heerebeek, N. Hamdani, M. L. Handoko, I. FalcaoPires, R. J. Musters, K. Kupreishvili, et al. Diastolic Stiffness of the Failing Diabetic Heart, Circulation, 2008, 117(1), 4351.

47 A. Borbély, J. van der Velden, Z. Papp, J. G. F. Bronzwaer, I. Edes, G. J. M. Stienen, et al. Cardiomyocyte Stiffness in Diastolic Heart Failure, Circulation, 2005, 111(6), 774-781.

48 J. Liang, B. Huang, G. Yuan, Y. Chen, F. Liang, H. Zeng, et al. Stretch-activated channel Piezo1 is up-regulated in failure heart and cardiomyocyte stimulated by AngII, Am. J. Transl. Res., 2017, 9(6), 2945-2955.

49 T. E. Walters, G. Lee, S. Spence, M. Larobina, V. Atkinson, P. Antippa, et al. Acute Atrial Stretch Results in Conduction Slowing and Complex Signals at the Pulmonary Vein to Left Atrial Junction, Circ.: Arrhythmia Electrophysiol., 2014, 7(6), 1189-1197.

50 B. John, M. K. Stiles, P. Kuklik, A. G. Brooks, S. T. Chandy, J. M. Kalman, et al. Reverse Remodeling of the Atria After Treatment of Chronic Stretch in Humans, J. Am. Coll. Cardiol., 2010, 55(12), 1217-1226.

51 J. I. Vandenberg, S. A. Rees, A. R. Wright and T. Powell, Cell swelling and ion transport pathways in cardiac myocytes, Cardiovasc. Res., 1996, 32, 85-97.

52 M. R. Franz, D. Burkhoff, D. T. Yue and K. Sagawa, Mechanically induced action potential changes and arrhythmia in isolated and in situ canine hearts, Cardiovasc. Res., 1989, 23(3), 213-223.

53 A. Salameh, S. Karl, H. Djilali, S. Dhein, J. Janousek and I. Daehnert, Opposing and synergistic effects of cyclic mechanical stretch and $\alpha$ - or $\beta$-adrenergic stimulation on the cardiac gap junction protein $\mathrm{Cx} 43$, Pharmacol. Res., 2010, 62(6), 506-513.
54 R. M. A. Van De Wal, H. W. M. Plokker, D. J. A. Lok, F. Boomsma, F. A. L. Van Der Horst, D. J. Van Veldhuisen, et al. Determinants of increased angiotensin II levels in severe chronic heart failure patients despite ACE inhibition, Int. J. Cardiol., 2006, 106(3), 367-372.

55 R. J. MacFadyen, A. F. C. Lee, J. J. Morton, S. D. Pringle and A. D. Struthers, How often are angiotensin II and aldosterone concentrations raised during chronic ACE inhibitor treatment in cardiac failure?, Heart, 1999, 82(1), 57-61.

56 M. C. Lampi and C. A. Reinhart-King, Targeting extracellular matrix stiffness to attenuate disease: From molecular mechanisms to clinical trials, Sci. Transl. Med., 2018, 10(422), eaao0475.

57 M. Sun, G. Chi, J. Xu, Y. Tan, J. Xu, S. Lv, et al. Extracellular matrix stiffness controls osteogenic differentiation of mesenchymal stem cells mediated by integrin $\alpha 5$, Stem Cell Res. Ther., 2018, 9(1), 52.

58 V. F. Fiore, S. S. Wong, C. Tran, C. Tan, W. Xu, T. Sulchek, et al. $\alpha \mathrm{v} \beta 3$ Integrin drives fibroblast contraction and strain stiffening of soft provisional matrix during progressive fibrosis, JCI Insight, 2018, 3(20), e97597.

59 R. R. Chaturvedi, T. Herron, R. Simmons, D. Shore, P. Kumar, B. Sethia, et al. Passive stiffness of myocardium from congenital heart disease and implications for diastole, Circulation, 2010, 121(8), 979-988.

60 R. Yamasaki, Y. Wu, M. McNabb, M. Greaser, S. Labeit and H. Granzier, Protein kinase A phosphorylates titin's cardiac-specific N2B domain and reduces passive tension in rat cardiac myocytes, Circ. Res., 2002, 90(11), 11811188.

61 N. Hamdani, K. G. Bishu, M. Von Frieling-Salewsky, M. M. Redfield and W. A. Linke, Deranged myofilament phosphorylation and function in experimental heart failure with preserved ejection fraction, Cardiovasc. Res., 2013, 97, 464-471.

62 W. Sumita Yoshikawa, K. Nakamura, D. Miura, J. Shimizu, K. Hashimoto, N. Kataoka, et al. Increased passive stiffness of cardiomyocytes in the transverse direction and residual actin and myosin cross-bridge formation in hypertrophied rat hearts induced by chronic $\beta$-adrenergic stimulation, Circ. J., 2013, 77(3), 741-748.

63 J. Chen, J.-K. Chen, E. G. Neilson and R. C. Harris, Role of EGF Receptor Activation in Angiotensin II-Induced Renal Epithelial Cell Hypertrophy, J. Am. Soc. Nephrol., 2006, 17(6), 1615-1623.

64 J. M. Siddesha, A. J. Valente, S. S. V. P. Sakamuri, T. Yoshida, J. D. Gardner, N. Somanna, et al. Angiotensin II stimulates cardiac fibroblast migration via the differential regulation of matrixins and RECK, J. Mol. Cell. Cardiol., 2013, 65, 9-18.

65 A. Hashimoto-Komatsu, T. Hirase, M. Asaka and K. Node, Angiotensin II induces microtubule reorganization mediated by a deacetylase SIRT2 in endothelial cells, Hypertens. Res., 2011, 34(8), 949-956.

66 A. Zeidan, S. Javadov and M. Karmazyn, Essential role of Rho/ROCK-dependent processes and actin dynamics in 
mediating leptin-induced hypertrophy in rat neonatal ventricular myocytes, Cardiovasc. Res., 2006, 72(1), 101-111.

67 Y. Wen, C. H. Eng, J. Schmoranzer, N. Cabrera-Poch, E. J. S. Morris, M. Chen, et al. EB1 and APC bind to mDia to stabilize microtubules downstream of Rho and promote cell migration, Nat. Cell Biol., 2004, 6(9), 820-830.

68 G. G. Gundersen, Y. Wen, C. H. Eng, J. Schmoranzer, N. Cabrera-Poch, E. J. S. Morris, et al. Regulation of microtubules by Rho GTPases in migrating cells, Novartis Found. Symp., 2005, 269, 106-116. discussion 116-26, 223-30.

69 A. Takesono, S. J. Heasman, B. Wojciak-Stothard, R. Garg and A. J. Ridley, Microtubules Regulate Migratory Polarity through Rho/ROCK Signaling in T Cells. Rappoport JZ, editor, PLoS One, 2010, 5(1), e8774.

70 T. Enomoto, Microtubule disruption induces the formation of actin stress fibers and focal adhesions in cultured cells: possible involvement of the rho signal cascade, Cell Struct. Funct., 1996, 21(5), 317-326.
71 S. Kadir, J. W. Astin, L. Tahtamouni, P. Martin and C. D. Nobes, Microtubule remodelling is required for the front-rear polarity switch during contact inhibition of locomotion, J. Cell Sci., 2011, 124(Pt 15), 26422653.

72 M. R. Zile, M. Koide, H. Sato, Y. Ishiguro, C. H. Conrad, J. M. Buckley, et al. Role of microtubules in the contractile dysfunction of hypertrophied myocardium, J. Am. Coll. Cardiol., 1999, 33(1), 250-260.

73 J. L. Sanchez-Alonso, A. Bhargava, T. O’Hara, A. V. Glukhov, S. Schobesberger, N. Bhogal, et al. Microdomain-Specific Modulation of L-Type Calcium Channels Leads to Triggered Ventricular Arrhythmia in Heart Failure, Circ. Res., 2016, 119(8), 944-955.

74 Y. Chen, G. B. Sukhorukov and P. Novak, Visualising nanoscale restructuring of a cellular membrane triggered by polyelectrolyte microcapsules, Nanoscale, 2018, 10(35), 16902-16910. 